\title{
All Solid State Reference Electrodes
}

\author{
Agata Michalska, Anna Kisiel, Krzysztof Maksymiuk \\ Faculty of Chemistry, Warsaw University, Pasteura 1, 02-093 Warsaw, Poland \\ agatam@chem.uw.edu.pl
}

\begin{abstract}
The important part of electrochemical measuring set up is a reference electrode, which is responsible for assuring constant potential during measurement. In conventional arrangement of reference electrode a liquid phase of concentrated electrolyte plays important role. The requirements for small, disposable electrochemical systems set the necessity of elimination of the liquid phase from its counter parts, including the reference electrode. This task is especially challenging if the potentiometric applications are envisaged, when high stability of reference element potentials in time is necessary. The composition of the reference membrane need to be tailored to show (ideally) no preference to any ion present in solution and to be relatively resistive to its composition changes. The approach we have adapted is based on incorporation of both cation- and ion-exchanger in the membrane phase, to assure balanced exchange of ions present in solution. The stability of potential is assured by the presence of silver/ silver chloride system accompanied by the solid potassium chloride, both dispersed in the polymeric membrane phase. The composition used was optimized with respect of membrane material applied resulting in highly stable potentials in time, independently of composition of the sample.
\end{abstract}

Key words: all-solid-state reference electrodes, reference electrode, ion-selective electrodes, all-solid-state potentiometric sensors

\section{Introduction}

In recent years a pronounced emphasis in research related to electroanalysis is on applications, driving interest in miniaturized, disposable electrochemical detections systems tailored for specific applications. In principle such systems usually consist of at least indicator or working electrode (differently called depending if it is current based or potentiometric measurements) and the reference electrode.

Miniaturization of working/ indicator electrode nowadays can be easily achieved. Disposable electrodes, e.g. prepared using screen printing approach and intended for current-based electrochemical techniques can be tailor made or purchased.

However, acknowledging the fact that typical reference electrode e.g. silver chloride one, contains a liquid, concentrated electrolyte solution, makes miniaturization and disposable use quite challenging

Thus, in electrochemical current based techniques for small, single-use, solution-free setups e.g. disposable, usually systems based on silver or silver/ silver chloride screen printed layers are used, without any additional outer coating layer [1]. Such electrodes are printed to make customized, optimized configuration of electrodes for certain applications. The main concern is in reproducibility of obtained sets, including printed reference element. Since the disposable applications are intended, this issue is more important than long-term stability of potential of reference element. In fact, the stability of potential in time is hardly ever studied for these type reference elements intended e.g. for amperometric applications [1].

On the contrary, if potentiometric applications are envisaged, elimination of the liquid phase does not abolish the requirement of long term stability of reference electrode potentials. In fact, this requirement is vitally important one, making elimination of electrolyte phase from the reference electrode challenging, yet practically oriented research topic.

\section{All-solid-state arrangement}

All-solid-state electrode concept is not a new issue in potentiometry. The basic tool for potentiometric analysis is ion-selective 
electrode, analytical signal of this sensor is generated on membrane separating the internal solution and the sample. Presence of internal solution in the sensor among others sets limitation on its applicability (e.g. temperature range, position in the system), as well as requires maintenance. Thus for quite a while now the research interest was on finding solution allowing elimination of internal solution without compromising sensor performance, including stability of potentials readings $[2,3]$. Currently, there is clear agreement in the field that the best option to obtain solution free arrangement is to apply a transducer layer between the electronically conducting (e.g. metallic or glassy carbon) support and ionselective membrane, Fig 1. The best suited materials to be applied as transducers seem to be conducting polymers, among those recently the preference is given to poly(3octylthiophene) [2,3]. Thus obtained arrangement is called all-solid-state electrode, Fig 1.

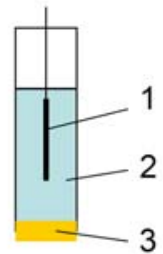

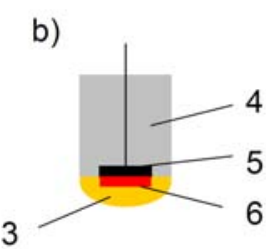

Fig. 1. Schematic representation of a) typical, i.e. of internal solution containing potentiometric electrode, b) all-solid-state arrangement; 1) internal reference electrode (typically $\mathrm{Ag} / \mathrm{AgCl}$ ), 2) internal solution (containing both analyte ions and $\mathrm{Cl}$ anions), 3) ion-selective membrane (e.g. poly(vinyl chloride) or polyacrylate), 4) electrode body, 5) substrate electrode (typically glassy carbon or Pt), 6) transducer layer (typically conducting polymer, e.g. poly(3-octylthiophene).

This approach can be also adapted for reference electrode, however introduction of transducer as such is solving just a part of the problem. The most important issue in the case of construction of reference element is composing a reference membrane assuring stable and reproducible potential of this part of electrochemical set up. Ideally, it would be convenient to prepare the reference membrane using the same approach/ materials which are used to prepare ion-selective membrane, to be able to relatively easily produce sets of electrodes. Nevertheless, such approach is limiting the samples that can be used to aqueous ones, it is most often sufficient as ionselective electrodes operation is also limited to above conditions.

\section{Composing reference membrane}

Typical ion-selective membranes are tailor made to show high preference for analyte ion over other constituents of sample solution, both of the same charge and opposite one. Thus, the ion-selective membrane contains apart from either cation- or anion- exchanging sites specific ionophore, all dispersed in synthetic matrix - typically plasticized poly(vinyl chloride) or polyacrylate.

The key issue while composing reference membrane is to assure no preference for cation and anion exchange between the membrane and solution. Moreover, the constituents of the membrane need to be compatible with the membrane matrix applied. Last but not the least, the membrane has to be able to exhibit constant potential for some time (days/ weeks), irrespectively for solution it is in contact with.

Thus the composition of our reference membrane was containing equimolar mixture of cation- and anion-exchanger, assuring balanced exchange of anions and cations between membrane and solution $[4,5,6,7]$. The stability of potentials was achieved due to introduction to the membrane of fine silver/ silver chloride powder and solid potassium chloride, Fig 2.

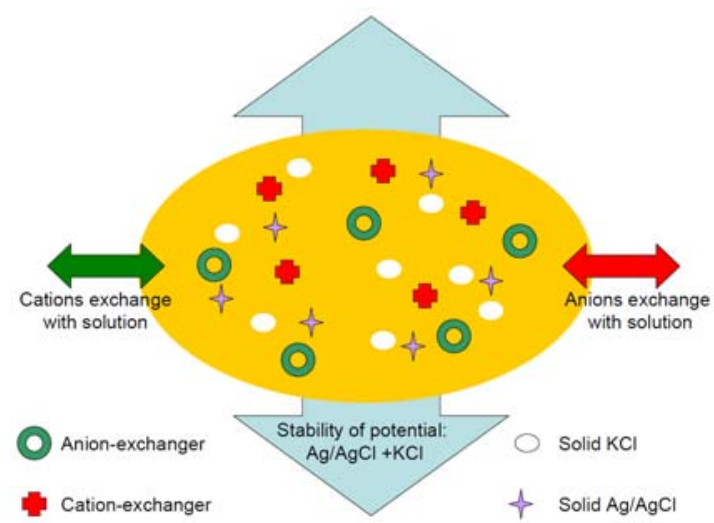

Fig. 2. Schematic representation of applied composition of reference membrane

\section{Performance of all-solid-state reference electrodes}

Thus obtained membrane was applied on the top of supporting electrode covered with transducer layer (similarly as usually done for indicator ion-selective electrodes). The appropriate pretreatment protocol was required to assure stable potentiometric responses of the reference electrode [4-7].

The applicability of proposed system was verified under different conditions, including 
varying composition of tested samples as well as changing conditions of experiments from potentiometry to current based electrochemical experiments.

The slope of dependences of potentials of proposed reference electrodes vs. logarithm of activity of solution recorded for poly(vinyl chloride) membrane matrix material was far below $1 \mathrm{mV} / \mathrm{dec}$, within the range from 0.1 to $10^{-5} \mathrm{M}$, the same within the range of experimental error (c.a. $1 \mathrm{mV}$ ) were recorded for model electrolytes tested (including $\mathrm{KCl}$, $\mathrm{NaCl}, \mathrm{NaNO}_{3}$ ) [4]. Thus obtained reference electrode was insensitive to $\mathrm{pH}$ changes within the broad range [4].

Equally good performance was characteristic also for other membrane material type, i.e. poly(n-butyl acrylate) [5]. Moreover, in the latter case the insensitivity of potential of tested electrodes for the presence of redox system in the sample, as well as presence of oxygen or nitrogen in the solution was confirmed [5]. Introduction of a model surfactant to solution, even at $10^{-3} \mathrm{M}$ concentration was not affecting potential values recorded while the activity of electrolyte was varied [5], both for polyacrylate and poly(vinyl chloride) membranes matrix, Fig 3. Moreover, linear calibration lines were recorded for potassium indicator electrode with typical detection limit close to $10^{-7} \mathrm{M}$, using above described reference element [5]. The obtained low value of detection limit of potassium selective electrode is clearly showing that there is no major leakage of potassium ions (or $\mathrm{KCl}$ ) from the membrane phase, which is beneficial for potential application of the reference electrode. Moreover, during overnight continuous experiment in $0.1 \mathrm{M} \mathrm{KCl}$ solutions the drift observed was in each case $<0.2 \mathrm{mV} / \mathrm{h}$; clearly showing excellent performance. 5 hours long experiments performed in stirred $0.1 \mathrm{M} \mathrm{KCl}$ solution, i.e. in conditions promoting deterioration of the reference electrode, observed stability of potential was slightly higher but still lower than $1.5 \mathrm{mV} / \mathrm{h}$.

Further studies were performed for extremely simple set up proposed by us, called all-plastic electrodes $[8,9]$ - where a conducting polymer is used both as transducer and electrical lead. Thus obtained reference electrodes were showing stable potentials in various electrolytes, which is highly promising for application in simple potentiometric setups, Fig 4. Using this approach we were able to prepare electrochemical set up consisting of indicator and reference electrode located together on a plastic foil strip [6]. Despite the simplicity of this arrangement, outstanding performance of model sensor - calcium or lead selective electrode - were obtained when tested against proposed all-solid state reference electrode.

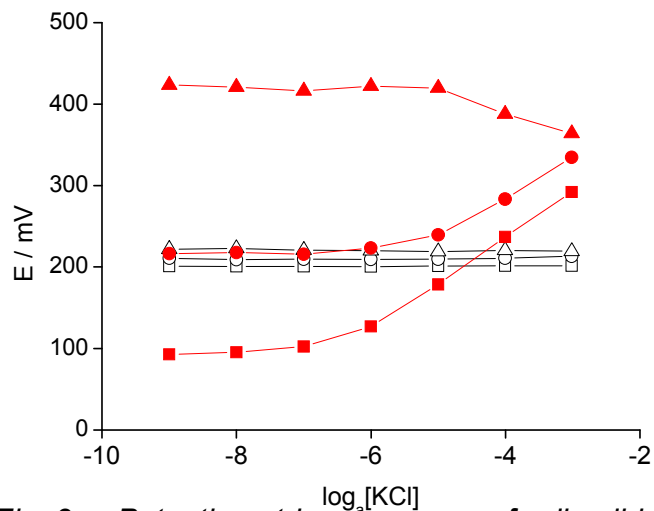

Fig. 3. Potentiometric reponses of all-solid state reference electrode with poly(vinyl chloride) based membrane (tested against classical reference electrode) recorded in $\mathrm{KCl}$ of different activities in the absence ( $\square$ ) and in the presence of different background concentrations: (०) $10^{-4} \mathrm{M}$ or $(\triangle) \quad 10^{-3} \mathrm{M}$ of model surfactant, trimethylhexylammonium chloride. Red symbols refer to all-solid-state indicator, potassium selective electrode tested against classical reference electrode and clearly show strong deteriorating effect of surfactant on recorded potentials.

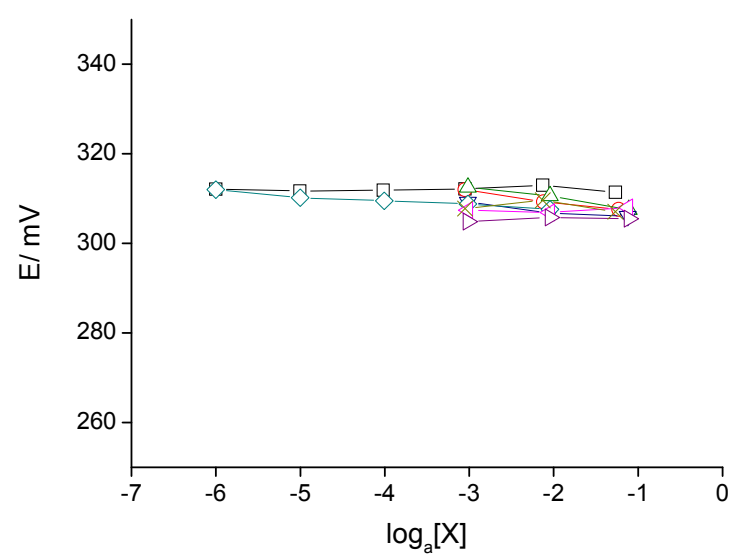

Fig. 4. Potentiometric reponses of all-plastic allsolid-state reference electrode with poly(vinyl chloride) based membrane (tested against classical reference electrode) subsequently in various model electrolytes and mean potentials recorded $\pm S D$ : (口) $\mathrm{CaCl}_{2} \mathrm{E}=312.1 \pm 0.6 \mathrm{mV}$, (०) $\mathrm{MgCl}_{2} E=304.5 \pm$ $0.6 \mathrm{mV},(\triangle) \mathrm{NaCl} E=304.9 \pm 0.8 \mathrm{mV},(\nabla) \mathrm{KCl} E=$ $303.9 \pm 1.3 \mathrm{mV},(\diamond) \mathrm{Pb}\left(\mathrm{NO}_{3}\right)_{2} \quad E=305.5 \pm 1.3 \mathrm{mV}$, $(\triangleleft) \mathrm{KCl} E=308.1 \pm 1.1 \mathrm{mV},(\triangleright) \mathrm{NaCl} E=306.0 \pm$ $0.8 \mathrm{mV},(x) \mathrm{LiCl} E=307.4 \pm 1.8 \mathrm{mV}$.

Our recent research were focused on incorporation of micro- and nanostructures for the benefit of proposed reference electrodes. Results obtained clearly show that poly(3-octylthiophene) can be easily replaced by other type transducers, moreover, some 
variation of the membrane matrix are not resulting in deterioration of the reference electrode performance [7]. Introduction of polymeric microspheres loaded with $\mathrm{KCl}$ or containing silver/ silver chloride can be advantageous for some polyarylate membranes compositions, especially those prepared from solution processable polymers [10]. Thus prepared reference electrodes were much improved, compared to systems using just solution processable polyacrylate as membrane material [10]. Also introduction of conducting polymer nanocapsules to the reference membrane composition was tested and found promising alternative for further improvements of internal solution free reference elements [11].

The research on all-solid-state reference electrodes are continued in our laboratory.

\section{Acknowledgements}

Financial support from National Centre of Science (NCN, Poland), project N N204 247640 , in the years 2011-2014, is gratefully acknowledged.

\section{References}

[1] A. Michalska, A. Kisiel, K. Maksymiuk, Handbook of Reference Electrodes, eds. G. Inzelt, A. Lewenstam, F. Scholz, Springer, in press.

[2] A. Michalska, Anal. Bioanal. Chem., 384 391- 406 (2006)

[3] J. Bobacka, Electroanalysis, 18, 7 - 18 (2006)

[4] A. Kisiel, H. Marcisz, A. Michalska, K. Maksymiuk, Analyst, 130, 1655 -1662 (2005)

[5] A. Kisiel, A. Michalska, K. Maksymiuk, E. A. H. Hall, Electroanalysis, 20, $318-323$ (2008)

[6] A. Kisiel, A. Michalska, A. Maksymiuk, Bioelectrochem, 71, 75 - 80 (2007)

[7] F. X. Rius-Ruiz, A. Kisiel, A. Michalska, K. Maksymiuk, J. Riu, F. X. Rius, Anal. Bioanal. Chem., 399, 3613-3622 (2011)

[8] A. Michalska, K. Maksymiuk, Anal. Chim. Acta, 523, 97 - 105 (2004)

[9] A. Michalska, M. Ocypa, K. Maksymiuk, Electroanalysis, 17, 327 - 333 (2005)

[10] A. Kisiel, M. Donten, J. Mieczkowski, F. X. Rius-Ruiz, K. Maksymiuk, A. Michalska, Analyst, 135, 2420-2425 (2010)

[11] A. Kisiel, K. Kijewska, M. Mazur, K. Maksymiuk, A. Michalska, Electroanalysis, 24, 165 - 172 (2012) 